\title{
QUADRANGLE GROUPS INCLUSIONS
}

\author{
ANTÓNIO BREDA D'AZEVEDO, DOMENICO A. CATALANO, JÁN KARABÁŠ, \\ AND ROMAN NEDELA
}

\begin{abstract}
In this paper we generalise Singerman's results on triangle group inclusions to the broader class of generalised quadrangle groups, that is, Fuchsian groups with three or four elliptic generators. For any possible inclusion $P<Q$ we also give the number of non-conjugate subgroups of $Q$ isomorphic to $P$.
\end{abstract}

\section{INTRODUCTION}

A finitely generated discrete subgroup of $\operatorname{PSL}(2, \mathbf{R})$, the group of conformal homeomorphisms of the upper half-plane $\mathbb{H}$, is called a Fuchsian group. Here we are interested only on the generalised quadrangle groups, that is, the Fuchsian groups $Q$ which have a presentation of the form

$$
Q(k, l, m, n)=\left\langle x, y, z, w \mid x^{k}=y^{l}=z^{m}=w^{n}=x y z w=1\right\rangle,
$$

for some positive integers $k, l, m$ and $n$. Since any Fuchsian group $\Gamma$ acts properly discontinuously on $\mathbb{H}$, any orbit $\Gamma z(z \in \mathbb{H})$ is a discrete subset of $\mathbb{H}$, which means that if $\Gamma z$ has a limit point $z_{0}$, then $z_{0} \in \mathbf{R} \cup\{\infty\}$. The collection $L(\Gamma)$ of all possible limit points of the orbits $\Gamma z, z \in \mathbb{H}$, is a subset of $\mathbf{R} \cup\{\infty\}$ satisfying one of the following properties:

(a) $L(\Gamma)$ has at most two points, or

(b) $L(\Gamma)=\mathbf{R} \cup\{\infty\}$, or

(c) $L(\Gamma)$ is a perfect nowhere-dense subset of $\mathbf{R} \cup\{\infty\}$.

Fuchsian groups satisfying (a) have either presentation $\left\langle x, y, p \mid x^{2}=y^{2}=x y p=1\right\rangle$, which is not a generalised quadrangle group, or are cyclic. Cyclic groups are not of our interest. Fuchsian groups satisfying (b) are said to be of the first kind. Their fundamental regions are of finite volume and, in the particular case of a generalised quadrangle group $Q(k, l, m, n)$, the volume is given by:

$$
\mu(Q(k, l, m, n))=2 \pi\left(2-\left(\frac{1}{k}+\frac{1}{l}+\frac{1}{m}+\frac{1}{n}\right)\right)>0 .
$$

Certainly this means that $\Sigma=\frac{1}{k}+\frac{1}{l}+\frac{1}{m}+\frac{1}{n}<2$. Fuchsian groups of the second kind are those satisfying (c); they have fundamental regions of infinite volume, and so not focused in this paper. In [13] Singerman classifies the normal inclusions between Fuchsian groups of the first kind having the same Teichmüller space dimension (that is, with the same sum $g+n$, where $g$ is the signature's genus and $n$ is the number of generators). Concerning non-normal inclusions, Singerman only classifies, in the same paper, the inclusions between triangle groups (non-cyclic Fuchsian groups with zero Teichmüller space dimension). More details about Fuchsian groups can be found in [10].

By (2) at most one of $\{k, l, m, n\}$ can be equal to one; if such case happens, then $Q(k, l, m, n)$ is a triangle group, otherwise it is a quadrangle group. Thus

2010 Mathematics Subject Classification. 20H10, 05C25, 20F65, 05C10, 57M60.

Key words and phrases. Fuchsian groups inclusions, constellations, dessin d'enfants. 
triangle groups $\Delta(l, m, n)$ are regarded as particular cases of generalised quadrangle groups. In this paper we extend Singerman's results about inclusions between triangle groups (see [13]) to inclusions between generalised quadrangle groups. Triangle group inclusions have been useful in the context of Riemann surfaces to define transformations between "Riemann hypermaps" ${ }^{1}$ preserving the Riemann surface (see [15], [7] and [3]).

From now on, by a generalised quadrangle group we will always mean a Fuchsian group of the first kind with a presentation of the form (1). Our main result can be stated as follows:

Theorem (main result). The finite index inclusions between generalised quadrangle groups $P=Q(p, q, r, s)$ and $Q=Q(k, l, m, n)$ are grouped in 63 infinite families, described in Tables 1,3,5,7 and 380 single inclusions, called sporadic, described in Tables 2,4,6,8.

All the tables exhibit the following information:

- the index $N$ of the inclusion (first column),

- $(p, q, r, s)$ and $(k, l, m, n)$ (second and third column),

- the passport $\left(\lambda\left(\pi_{x}\right), \lambda\left(\pi_{y}\right), \lambda\left(\pi_{z}\right), \lambda\left(\pi_{w}\right)\right)$ of the inclusion, that is, the cycle structures of the generators $x, y, z, w$ of $Q$ regarded as permutations $\pi_{x}, \pi_{y}, \pi_{z}, \pi_{w}$ of the $N$ cosets of $P$ in $Q$ (fourth column),

- the number \# of realisations, that is, the number of non-conjugate subgroups of $Q$ isomorphic to $P$ (last column).

As a corollary we have the following:

Corollary. Let $P<Q$ be an inclusion of index $N$ between generalised quadrangle groups. Then

- $N \leq 60$, and $N=60$ for the six realisations of $P=Q(7,7,7,7)<Q=$ $Q(1,2,3,7)=\Delta(2,3,7)$ (see Table 4). Note that Lemma 2.4 gives $N<84$ (hence Riemann-Hurwitz bound $N=83$ is not attained).

- If $P<Q$ belongs to a family (see Tables 1, 3, 5 and 7), then $N \leq 12$ and $N=12$ if and only if $P<Q$ belongs to one of the six families given in Table 3.

- The maximal number of realisations is 16 attained by $P=Q(3,3,7,7)<$ $Q=Q(1,2,3,7)=\Delta(2,3,7)$ of index 44 (see Table 8$)$.

More details as well as representations of generalised quadrangle group inclusions by pictures (spherical dessin d'enfants) can be found in the paper [4] and on the web page [5].

\section{Preliminaries}

The following claims are consequences of well-known properties of Fuchsian groups.

Lemma 2.1. The generators $x, y, z$ and $w$ of a generalised quadrangle group $Q(k, l, m, n)$ have order $k, l, m$ and $n$, respectively.

Proof. Straightforward from the presentation (1), since $Q(k, l, m, n)$ is the quotient of the free product $F$ of the cyclic groups $C_{k}=\left\langle x \mid x^{k}=1\right\rangle, C_{l}=\left\langle y \mid y^{l}=1\right\rangle$, $C_{m}=\left\langle z \mid z^{m}=1\right\rangle$ and $C_{n}=\left\langle w \mid w^{n}=1\right\rangle$ by the normal closure in $F$ of the cyclic

\footnotetext{
${ }^{1}$ We mean hypermaps with underlying Riemann surfaces whose projection to the sphere induced by the hypermap projection to the trivial spherical hypermap is a Belyu function - every hypermap actually induces a "Riemann hypermap" and conversely (see [14]).
} 
group generated by $x y z w$, which has trivial intersection with any of the cyclic groups $C_{k}, C_{l}, C_{m}$ and $C_{n}$.

Lemma 2.2. For any permutation $\sigma$ of degree 4 , the group

$$
Q\left(m_{\sigma(1)}, m_{\sigma(2)}, m_{\sigma(3)}, m_{\sigma(4)}\right)
$$

is isomorphic to $Q\left(m_{1}, m_{2}, m_{3}, m_{4}\right)$.

Proof. As the permutations $(1,2)$ and $(2,3,4)$ generate the symmetric group of degree 4 , it is enough to show that $Q\left(m_{2}, m_{1}, m_{3}, m_{4}\right)$ and $Q\left(m_{1}, m_{3}, m_{4}, m_{2}\right)$ are isomorphic to $Q\left(m_{1}, m_{2}, m_{3}, m_{4}\right)$. The mapping

$$
x \mapsto x y x^{-1}, \quad y \mapsto x, \quad z \mapsto z, \quad w \mapsto w
$$

extends to a group isomorphism from $Q\left(m_{2}, m_{1}, m_{3}, m_{4}\right)$ to $Q\left(m_{1}, m_{2}, m_{3}, m_{4}\right)$, since it corresponds to a Tietze transformation of the first presentation giving the second as a result. Analogously, the mapping

$$
x \mapsto x, \quad y \mapsto w, \quad z \mapsto w^{-1} y w, \quad w \mapsto w^{-1} z w
$$

extends to an isomorphism from $Q\left(m_{1}, m_{3}, m_{4}, m_{2}\right)$ to $Q\left(m_{1}, m_{2}, m_{3}, m_{4}\right)$.

By means of Lemma 2.2, we can always assume that $k \leq l \leq m \leq n$ for representatives $Q(k, l, m, n)$ of isomorphism classes of generalised quadrangle groups. For $k=1$ we have that $Q(1, l, m, n)$ is the triangle group $\Delta(l, m, n)$.

From now on, let $P=Q(p, q, r, s)$ be a generalised quadrangle group with generators $a, b, c, d$ and presentation

$$
\left\langle a, b, c, d \mid a^{p}=b^{q}=c^{r}=d^{s}=a b c d=1\right\rangle
$$

and let $Q=Q(k, l, m, n)$ be a generalised quadrangle group with generators $x, y, z, w$ and presentation (1). Suppose that $P$ is a subgroup of index $N>1$ of $Q$ and write $P<_{N} Q$. Then, by means of the Riemann-Hurwitz formula, we have

$$
N=\frac{\mu(P)}{\mu(Q)}=\frac{2-\left(\frac{1}{p}+\frac{1}{q}+\frac{1}{r}+\frac{1}{s}\right)}{2-\left(\frac{1}{k}+\frac{1}{l}+\frac{1}{m}+\frac{1}{n}\right)} .
$$

Remark 2.3. Setting $\Sigma^{\prime}=\frac{1}{p}+\frac{1}{q}+\frac{1}{r}+\frac{1}{s}$ and $\Sigma=\frac{1}{k}+\frac{1}{l}+\frac{1}{m}+\frac{1}{n}$, we express (4) by $N=\frac{2-\Sigma^{\prime}}{2-\Sigma}$. Note that $\Sigma<2 \Leftrightarrow \Sigma^{\prime}<2$.

Lemma 2.4 (Riemann-Hurwitz bound). If $P<_{N} Q$ then $N<84$.

Proof. According to (4) we have $N<\frac{2}{2-\Sigma}$. As $\Sigma \leq \frac{1}{1}+\frac{1}{2}+\frac{1}{3}+\frac{1}{7}=\frac{83}{42}$ for integers $k, l, m, n$ satisfying $\frac{1}{k}+\frac{1}{l}+\frac{1}{m}+\frac{1}{n}<2$, we have $N<\frac{2}{2-\frac{83}{42}}=84$.

Lemma 2.5. If $P<_{N} Q$ and $Q=Q(k, l, m, n)$ with $k \leq l \leq m \leq n$, then $k<4$ and if $k=3$, then $N=2$.

Proof. From (4) we have $N<\frac{2}{2-\Sigma}$ and therefore

$$
1 \leq 2-\frac{2}{N}<\Sigma
$$

As $\Sigma \leq \frac{4}{k}$ by assumption, from (5) we conclude that $1<\frac{4}{k}$, that is, $k<4$. Let now $k=3$. Then $\Sigma \leq \frac{1}{3}+\frac{3}{l} \leq \frac{4}{3}$ by assumption. Hence (5) implies $2-\frac{2}{N}<\frac{4}{3}$, that is, $N<3$.

Looking at Theorem 1 of [13], which list all the normal inclusions between Fuchsian groups of the first kind that have the same Teichmüller space dimension, we found that $Q(m, m, n, n) \triangleleft_{2} Q(2,2, m, n)$ is the only index 2 family of inclusions between quadrangle groups. Hence we can state the following Proposition. 
Proposition 2.6. If $P<_{N} Q=Q(k, l, m, n)$ with $k \leq l \leq m \leq n$, then $k=1$ or $k=2$.

\section{QUADRANGLE GROUPS INCLUSIONS}

The following lemma will play an important rôle in our approach. It is a consequence of the following well-known facts (see [9] or [10], for instance):

(a) Elements of finite order in $\operatorname{PSL}(2, \mathbb{R})$ are elliptic;

(b) Any elliptic element of a Fuchsian group $\Gamma$ is conjugate (in $P S L(2, \mathbb{R})$ ) to a power of an elliptic generator of $\Gamma$.

Lemma 3.1. Any non-trivial element of finite order in $Q$ is conjugate to a power of one and only one generator of $Q$.

Let $g, h$ be elements of $P S L(2, \mathbb{R})$. We write $g \vdash h$ to mean that the element $g$ is conjugate to a power of $h$. Denoting by $|g|$ the order of $g$, if $g, h$ have finite order and $g \vdash h$, then $|g|$ divides $|h|$.

If $P<_{N} Q$, then $a, b, c, d$ are elements of finite order in $Q$ and Lemma 3.1 guaranties that for any $a_{j} \in\{a, b, c, d\}$ there is a unique $x_{i} \in\{x, y, z, w\}$ such that $a_{j} \vdash x_{i}$. Moreover, up to a permutation of $\{a, b, c, d\}$ and a permutation of $\{x, y, z, w\}$ (Lemma 2.2), one of the following cases occurs for $N>1$ :

Case 1. $a, b, c, d \vdash w$. (Then $p, q, r$ and $s$ divide $n$ ).

Case 2. $a \vdash z$ and $b, c, d \vdash w$. (Then $p$ divides $m$ and $q, r, s$ divide $n$.)

Case 3. $a, b \vdash z$ and $c, d \vdash w$. (Then $p, q$ divide $m$ and $r, s$ divide $n$.)

Case 4. $a \vdash y, b \vdash z$ and $c, d \vdash w$. (Then $p$ divides $l, q$ divides $m$ and $r, s$ divide n.)

Remark. It looks like that the case $a \vdash x, b \vdash y, c \vdash z$ and $d \vdash w$ is missing, however this case implies that $p$ divides $k, q$ divides $l, r$ divides $m$ and $s$ divides $n$, giving

$$
\Sigma=\frac{1}{k}+\frac{1}{l}+\frac{1}{m}+\frac{1}{n} \leq \frac{1}{p}+\frac{1}{q}+\frac{1}{r}+\frac{1}{s}=\Sigma^{\prime},
$$

and by (4) $N=\frac{2-\Sigma^{\prime}}{2-\Sigma} \leq 1$.

Given an inclusion $P<_{N} Q$ and a generator $x_{i}$ of $Q$, we denote by $\mathfrak{S}_{x_{i}}$ the set of elements $a_{j} \in\{a, b, c, d\}$ such that $a_{j} \vdash x_{i}$. Relatively to the above cases we have:

Case 1: $\mathfrak{S}_{x}=\mathfrak{S}_{y}=\mathfrak{S}_{z}=\emptyset$ and $\mathfrak{S}_{w}=\{a, b, c, d\}$.

Case 2: $\mathfrak{S}_{x}=\mathfrak{S}_{y}=\emptyset, \mathfrak{S}_{z}=\{a\}$ and $\mathfrak{S}_{w}=\{b, c, d\}$.

Case 3: $\mathfrak{S}_{x}=\mathfrak{S}_{y}=\emptyset, \mathfrak{S}_{z}=\{a, b\}$ and $\mathfrak{S}_{w}=\{c, d\}$.

Case 4: $\mathfrak{S}_{x}=\emptyset, \mathfrak{S}_{y}=\{a\}, \mathfrak{S}_{z}=\{b\}$ and $\mathfrak{S}_{w}=\{c, d\}$.

The following proposition characterises the orbits of the action of generators of $Q$ on the $N$ cosets of $P$ in $Q$ for $P<_{N} Q$. For its proof see Theorem 1 in [12].

Proposition 3.2 (Singerman). There is an inclusion $P<_{N} Q$ if and only if there is a permutation group $G$ transitive on $N$ points and an epimorphism $\pi: Q \rightarrow G$ such that for any $x_{i} \in\{x, y, z, w\}$ the permutation $\pi\left(x_{i}\right)$ satisfies the following property:

There is a one-to-one mapping $\gamma$ from the set of non-trivial elements of $\mathfrak{S}_{x_{i}}$ to the set of cycles of $\pi\left(x_{i}\right)$ of length less than $\left|x_{i}\right|$ such that any cycle $\gamma\left(a_{j}\right)$ has length $\frac{\left|x_{i}\right|}{\left|a_{j}\right|}$.

In the language of constellations (see [11], for instance), Proposition 3.2 says that any inclusion $P<_{N} Q$ gives rise to a 4-constellation $\left(\pi_{x}, \pi_{y}, \pi_{z}, \pi_{w}\right)$ of degree $N$ 
with passport $\left(\lambda\left(\pi_{x}\right), \lambda\left(\pi_{y}\right), \lambda\left(\pi_{z}\right), \lambda\left(\pi_{w}\right)\right)$, where

$$
\lambda\left(\pi_{x_{i}}\right)= \begin{cases}\left|x_{i}\right|^{\frac{N}{\left|x_{i}\right|}} & \text { if } \mathfrak{S}_{x_{i}}=\emptyset \\ \left.\frac{\left|x_{i}\right|}{\left|a_{1}\right|} \ldots \ldots\left|\frac{\left|x_{i}\right|}{\left|a_{\alpha}\right|} \cdot\right| x_{i}\right|^{\frac{N-\left(\frac{\left|x_{i}\right|}{\left|a_{1}\right|}+\cdots+\frac{\left|x_{i}\right|}{\left|a_{\alpha}\right|}\right)}{\left|x_{i}\right|}} & \text { if } \mathfrak{S}_{x_{i}}=\left\{a_{1}, \ldots, a_{\alpha}\right\} \neq \emptyset\end{cases}
$$

for any $x_{i} \in\{x, y, z, w\}$, with the convention that

$$
\begin{gathered}
\qquad \frac{\left|x_{i}\right|}{\left|a_{1}\right|} \cdots \frac{\left|x_{i}\right|}{\left|a_{\alpha}\right|} \cdot\left|x_{i}\right| \frac{N-\left(\frac{\left|x_{i}\right|}{\left|a_{1}\right|}+\cdots+\frac{\left|x_{i}\right|}{\left|a_{\alpha}\right|}\right)}{\left|x_{i}\right|}=\frac{\left|x_{i}\right|}{\left|a_{1}\right|} \cdots \frac{\left|x_{i}\right|}{\left|a_{\alpha}\right|} \\
\text { if } N-\left(\frac{\left|x_{i}\right|}{\left|a_{1}\right|}+\cdots+\frac{\left|x_{i}\right|}{\left|a_{\alpha}\right|}\right)=0 .
\end{gathered}
$$

Using Proposition 3.2 we can be more precise in each case.

Lemma 3.3 (Case 1). If $P<_{N} Q$ and $a, b, c, d \vdash w$, then

(i) $p, q, r, s$ divide $n$.

(ii) $k, l, m$ divide $N$.

(iii) $n$ divides $N-\left(\frac{n}{p}+\frac{n}{q}+\frac{n}{r}+\frac{n}{s}\right)$.

(iv) The equality (4) takes the following form

$$
2(N-1)-\frac{N}{k}-\frac{N}{l}-\frac{N}{m}=\frac{N-\left(\frac{n}{p}+\frac{n}{q}+\frac{n}{r}+\frac{n}{s}\right)}{n} .
$$

(v) There is a 4-constellation of degree $N$ and passport

$$
\left(k^{\frac{N}{k}}, l^{\frac{N}{l}}, m^{\frac{N}{m}}, \frac{n}{p} \cdot \frac{n}{q} \cdot \frac{n}{r} \cdot \frac{n}{s} \cdot n^{\frac{N-\left(\frac{n}{p}+\frac{n}{q}+\frac{n}{r}+\frac{n}{s}\right)}{n}}\right) .
$$

Lemma 3.4 (Case 2). If $P<_{N} Q, a \vdash z$ and $b, c, d \vdash w$, then

(i) $p$ divides $m$ and $q, r, s$ divide $n$.

(ii) $k, l$ divide $N$.

(iii) $m$ divides $N-\frac{m}{p}$ and $n$ divides $N-\left(\frac{n}{q}+\frac{n}{r}+\frac{n}{s}\right)$.

(iv) The equality (4) is equivalent to

$$
2(N-1)-\frac{N}{k}-\frac{N}{l}=\frac{N-\frac{m}{p}}{m}+\frac{N-\left(\frac{n}{q}+\frac{n}{r}+\frac{n}{s}\right)}{n} .
$$

(v) There is a 4-constellation of degree $N$ and passport

$$
\left(k^{\frac{N}{k}}, l^{\frac{N}{l}}, \frac{m}{p} \cdot m^{\frac{N-\frac{m}{p}}{m}}, \frac{n}{q} \cdot \frac{n}{r} \cdot \frac{n}{s} \cdot n^{\frac{N-\left(\frac{n}{q}+\frac{n}{r}+\frac{n}{s}\right)}{n}}\right) .
$$

Lemma 3.5 (Case 3). If $P<_{N} Q, a, b \vdash z$ and $c, d \vdash w$, then

(i) $p, q$ divide $m$ and $r, s$ divide $n$.

(ii) $k, l$ divide $N$.

(iii) $m$ divides $N-\left(\frac{m}{p}+\frac{m}{q}\right)$ and $n$ divides $N-\left(\frac{n}{r}+\frac{n}{s}\right)$.

(iv) The equality (4) is equivalent to

$$
2(N-1)-\frac{N}{k}-\frac{N}{l}=\frac{N-\left(\frac{m}{p}+\frac{m}{q}\right)}{m}+\frac{N-\left(\frac{n}{r}+\frac{n}{s}\right)}{n} .
$$

(v) There is a 4-constellation of degree $N$ and passport

$$
\left(k^{\frac{N}{k}}, l^{\frac{N}{l}}, \frac{m}{p} \cdot \frac{m}{q} \cdot m^{\frac{N-\left(\frac{m}{p}+\frac{m}{q}\right)}{m}}, \frac{n}{r} \cdot \frac{n}{s} \cdot n^{\frac{N-\left(\frac{n}{r}+\frac{n}{s}\right)}{n}}\right) .
$$

Lemma 3.6 (Case 4). If $P<_{N} Q, a \vdash y, b \vdash z$ and $c, d \vdash w$, then 
(i) $p$ divides $l, q$ divides $m$ and $r, s$ divide $n$.

(ii) $k$ divides $N$.

(iii) $l$ divides $N-\frac{l}{p}, m$ divides $N-\frac{m}{q}$ and $n$ divides $N-\left(\frac{n}{r}+\frac{n}{s}\right)$.

(iv) The equality (4) is equivalent to

$$
2(N-1)-\frac{N}{k}=\frac{N-\frac{l}{p}}{l}+\frac{N-\frac{m}{q}}{m}+\frac{N-\left(\frac{n}{r}+\frac{n}{s}\right)}{n} .
$$

(v) There is a 4-constellation of degree $N$ and passport

$$
\left(k^{\frac{N}{k}}, \frac{l}{p} \cdot l^{\frac{N-\frac{l}{p}}{l}}, \frac{m}{q} \cdot m^{\frac{N-\frac{m}{q}}{m}}, \frac{n}{r} \cdot \frac{n}{s} \cdot n^{\frac{N-\left(\frac{n}{r}+\frac{n}{s}\right)}{n}}\right) .
$$

\section{ReAlisation of inClusions}

In this section we describe how to obtain all the possible inclusions between generalised quadrangle groups. First, we solve the equality (iv) case by case (lemmas 3.3 to 3.6). Then, for each solution we construct the corresponding passport as a result of (v). Finally we exhibit a 4-constellation associated to each passport; by Proposition 3.2 it is enough to find for each inclusion $P<Q$ associated to a passport $\lambda$, a 4-constellation with passport $\lambda$. Actually we go well along in this step by computing all 4-constellations with passport $\lambda$. These give all non-conjugate subgroups of $Q$ of index $N$ isomorphic to $P$ (non-isomorphic 4-constellations).

As an example let us work out the Case 4 in details. When solving (iv) of Lemma 3.6 we reach the following two subcases:

(I) $2(N-1)-\frac{N}{k}=0$, which implies $N=2$ and $k=1$ and all summands on the right-hand side of (iv) are equal to zero.

(II) $2(N-1)-\frac{N}{k}>0$, which implies that at least one of the summands on the right-hand side of (iv) is greater than zero.

In subcase (I) we have $2 p=l, 2 q=m$, and $2=\frac{n}{r}+\frac{n}{s}$. Hence $(k, l, m, n)=$ $(1,2 p, 2 q, r)$ and $s=r$. This is a parametric $\left(\right.$ ordered $\left.^{2}\right)$ numerical solution which may give rise to a 3 -parametric family of normal inclusions

$$
Q(p, q, r, r) \triangleleft_{2} \Delta(2 p, 2 q, r) .
$$

According to $(\mathrm{v})$ of Lemma 3.6, any of these inclusions is realised provided that we are able to find a 4 -constellation $\left(\pi_{x}, \pi_{y}, \pi_{z}, \pi_{w}\right)$ with passport $\lambda=\left(1^{2}, 2,2,1^{2}\right)$. Remark that the passport is independent of the parameters. It is not complicated to see that $\left(\pi_{x}, \pi_{y}, \pi_{z}, \pi_{w}\right)=(1,(12),(12), 1)$, where $\pi_{x} \pi_{y} \pi_{z} \pi_{w}=1$, is a constellation associated to $\lambda$. This proves the existence of the inclusions (6). Moreover, there is no other constellation with passport $\lambda$ and, as $\pi_{x}=1$, we can reduce the 4 constellation $\left(\pi_{x}, \pi_{y}, \pi_{z}, \pi_{w}\right)$ to the 3 -constellation $\left(\pi_{y}, \pi_{z}, \pi_{w}\right)$ that we can depict as a spherical hypermap.

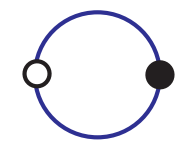

Figure 1: The hypermap (3-constellation) with passport $\left(2,2,1^{2}\right)$.

In subcase (II) some families of inclusions emerge when one or two of the righthand side summands in (iv) are equal to zero, resulting into 1- and 2-parametric

\footnotetext{
${ }^{2}$ Numerical solutions are taken up to permutations of $k, l, m, n$ and $p, q, r, s$.
} 
families:

1-parametric: (a1) $2(N-1)-\frac{N}{k}=\frac{N-\frac{m}{q}}{m}+\frac{N-\left(\frac{n}{r}+\frac{n}{s}\right)}{n}$ with only $\frac{N-\frac{l}{p}}{l}=0$.

(a2) $2(N-1)-\frac{N}{k}=\frac{N-\frac{l}{p}}{l}+\frac{N-\left(\frac{n}{r}+\frac{n}{s}\right)}{n}$ with only $\frac{N-\frac{m}{q}}{m}=0$.

(b) $2(N-1)-\frac{N}{k}=\frac{N-\frac{l}{p}}{l}+\frac{N-\frac{m}{q}}{m}$ with only $\frac{N-\left(\frac{n}{r}+\frac{n}{s}\right)}{n}=0$.

2-parametric: (a) $2(N-1)-\frac{N}{k}=\frac{N-\left(\frac{n}{r}+\frac{n}{s}\right)}{n}$ with $\frac{N-\frac{l}{p}}{l}=\frac{N-\frac{m}{q}}{m}=0$.

(b1) $2(N-1)-\frac{N}{k}=\frac{N-\frac{m}{q}}{m}$ with $\frac{N-\frac{l}{p}}{l}=\frac{N-\left(\frac{n}{r}+\frac{n}{s}\right)}{n}=0$.

(b2) $2(N-1)-\frac{N}{k}=\frac{N-\frac{l}{p}}{l}$ with $\frac{N-\frac{m}{q}}{m}=\frac{N-\left(\frac{n}{r}+\frac{n}{s}\right)}{n}=0$.

The 1-parametric equations (a1) and (a2) are the same up to transposition of $l$ and $m$. This equation give rise to two numerical solutions, both realisable, shown in lines 5 and 9 of Table 1 . The third equation (b) gives rise to 9 realisable numerical solutions (Table 1 , lines 4,6,7,8,10-14), two of them twice realisable.

The 2-parametric equation (a) has no numerical solution under the constrained (i)-(iii) of Lemma 3.6 and the assumption we made in (2). In fact, as

$$
N-2 \leq 2(N-1)-\frac{N}{k}=\frac{N-\left(\frac{n}{r}+\frac{n}{s}\right)}{n} \leq \frac{N-2}{n},
$$

subcase (II) forces $N$ to be greater than 2, giving $n=k=1$, in contradiction with (2). The two other equations (b1) and (b2), which are the same (up to transposition of $l$ and $m$ ), have two numerical solutions which are both once realisable (Table 1, lines 2 and 3 ).

If all three right-hand side summands of (iv) are non-zero, then we get 29 sporadic numerical solutions (i.e. non-parametric), 28 of which are realised by sporadic inclusions (Table 2).

\begin{tabular}{|r|r|l|c|c|}
\hline \hline$N$ & $(p, q, r, s)$ & $(k, l, m, n)$ & Passport & $\#$ \\
\hline \hline 2 & $(p, q, r, r)$ & $(1,2 p, 2 q, r)$ & $\left(1^{2}, 2,2,1^{2}\right)$ & 1 \\
2 & $(1, q, r, r)$ & $(1,2,2 q, r)$ & $\left(1^{2}, 2,2,1^{2}\right)$ & 1 \\
3 & $(2, q, r, 2 r)$ & $(1,2,3 q, 2 r)$ & $\left(1^{3}, 1.2,3,1.2\right)$ & 1 \\
3 & $(1,2, r, 2 r)$ & $(1,2,3,2 r)$ & $\left(1^{3}, 1.2,3,1.2\right)$ & 1 \\
4 & $(2,2,3, s)$ & $(1,2,3,4 s)$ & $\left(1^{4}, 1^{2} .2,1.3,4\right)$ & 1 \\
4 & $(3,3, r, 3 r)$ & $(1,3,3,3 r)$ & $\left(1^{4}, 1.3,1.3,1.3\right)$ & 1 \\
4 & $(1,3, r, 3 r)$ & $(1,2,3,3 r)$ & $\left(1^{4}, 2^{2}, 1.3,1.3\right)$ & 1 \\
4 & $(3,3, r, r)$ & $(1,3,3,2 r)$ & $\left(1^{4}, 1.3,1.3,2^{2}\right)$ & 1 \\
5 & $(2,3,3, s)$ & $(1,2,3,5 s)$ & $\left(1^{5}, 1.2^{2}, 1^{2} .3,5\right)$ & 1 \\
5 & $(2,4, r, 4 r)$ & $(1,2,4,4 r)$ & $\left(1^{5}, 1.2^{2}, 1.4,1.4\right)$ & 2 \\
5 & $(2,4,2 r, 3 r)$ & $(1,2,4,6 r)$ & $\left(1^{5}, 1.2^{2}, 1.4,2.3\right)$ & 1 \\
7 & $(2,3, r, 6 r)$ & $(1,2,3,6 r)$ & $\left(1^{7}, 1.2^{3}, 1.3^{2}, 1.6\right)$ & 2 \\
7 & $(2,3,2 r, 5 r)$ & $(1,2,3,10 r)$ & $\left(1^{7}, 1.2^{3}, 1.3^{2}, 2.5\right)$ & 1 \\
7 & $(2,3,3 r, 4 r)$ & $(1,2,3,12 r)$ & $\left(1^{7}, 1.2^{3}, 1.3^{2}, 3.4\right)$ & 1 \\
\hline \hline
\end{tabular}

TABLE 1. Families of inclusions, corresponding passports, and number of realisations in Case 4.

Tables 1 and 2 contain all the inclusions and the corresponding passports obtained in that case (Case 4). We also record the number of non-isomorphic 4constellation for each corresponding passport. These constellations were obtained in the following way: Given $Q=Q(k, l, m, n)$ we search trough all subgroups $P<_{N} Q$. Every subgroup $P$ gives rise to a homomorphism $\pi$, given by the action 
of $Q$ on the set of cosets of $P$. We then compute the passport of the 4-constellation $(\pi(x), \pi(y), \pi(z), \pi(w))$. If the obtained passport is included in the list of corresponding passports to numerical solutions for $Q(k, l, m, n)$, then $P<_{N} Q$ is realisable. This task can be accomplished by using computer algebra systems as GAP [6] or Magma [2].

\begin{tabular}{r|r|l|c|r|}
\hline \hline$N$ & $(p, q, r, s)$ & $(k, l, m, n)$ & Passport & $\#$ \\
\hline \hline 5 & $(3,3,4,4)$ & $(1,3,4,4)$ & $\left(1^{5}, 1^{2} .3,1.4,1.4\right)$ & 1 \\
6 & $(2,2,5,5)$ & $(1,2,5,5)$ & $\left(1^{6}, 1^{2} .2^{2}, 1.5,1.5\right)$ & 3 \\
6 & $(2,2,2,5)$ & $(1,2,4,5)$ & $\left(1^{6}, 1^{2} .2^{2}, 2.4,1.5\right)$ & 2 \\
6 & $(1,4,4,5)$ & $(1,2,4,5)$ & $\left(1^{6}, 2^{3}, 1^{2} .4,1.5\right)$ & 1 \\
7 & $(2,5,5,6)$ & $(1,2,5,6)$ & $\left(1^{7}, 1.2^{3}, 1^{2} .5,1.6\right)$ & 3 \\
7 & $(3,3,5,5)$ & $(1,3,3,5)$ & $\left(1^{7}, 1.3^{2}, 1.3^{2}, 1^{2} .5\right)$ & 1 \\
7 & $(2,2,4,6)$ & $(1,2,4,6)$ & $\left(1^{7}, 1.2^{3}, 1.2 .4,1.6\right)$ & 4 \\
7 & $(2,3,3,4)$ & $(1,3,3,4)$ & $\left(1^{7}, 1.3^{2}, 1.3^{2}, 1.2 .4\right)$ & 4 \\
8 & $(1,3,3,7)$ & $(1,2,3,7)$ & $\left(1^{8}, 2^{4}, 1^{2} .3^{2}, 1.7\right)$ & 1 \\
9 & $(2,4,7,7)$ & $(1,2,4,7)$ & $\left(1^{9}, 1.2^{4}, 1.4^{2}, 1^{2} .7\right)$ & 4 \\
9 & $(1,2,7,7)$ & $(1,2,3,7)$ & $\left(1^{9}, 1.2^{4}, 3^{3}, 1^{2} .7\right)$ & 1 \\
9 & $(2,3,4,6)$ & $(1,2,4,6)$ & $\left(1^{9}, 1.2^{4}, 1.4^{2}, 1.2 .6\right)$ & 4 \\
10 & $(2,2,3,9)$ & $(1,2,3,9)$ & $\left(1^{10}, 1^{2} .2^{4}, 1.3^{3}, 1.9\right)$ & 6 \\
10 & $(2,2,3,4)$ & $(1,2,3,8)$ & $\left(1^{10}, 1^{2} .2^{4}, 1.3^{3}, 2.8\right)$ & 3 \\
10 & $(3,3,4,4)$ & $(1,3,3,4)$ & $\left(1^{10}, 1.3^{3}, 1.3^{3}, 1^{2} .4^{2}\right)$ & 4 \\
10 & $(1,3,8,8)$ & $(1,2,3,8)$ & $\left(1^{10}, 2^{5}, 1.3^{3}, 1^{2} .8\right)$ & 1 \\
11 & $(2,3,3,10)$ & $(1,2,3,10)$ & $\left(1^{11}, 1.2^{5}, 1^{2} .3^{3}, 1.10\right)$ & 6 \\
11 & $(2,2,4,5)$ & $(1,2,4,5)$ & $\left(1^{11}, 1.2^{5}, 1.2 .4^{2}, 1.5^{2}\right)$ & 5 \\
13 & $(2,3,11,11)$ & $(1,2,3,11)$ & $\left(1^{13}, 1.2^{6}, 1.3^{4}, 1^{2} .11\right)$ & 6 \\
13 & $(2,3,5,10)$ & $(1,2,3,10)$ & $\left(1^{13}, 1.2^{6}, 1.3^{4}, 1.2 .10\right)$ & 6 \\
13 & $(2,3,3,9)$ & $(1,2,3,9)$ & $\left(1^{13}, 1.2^{6}, 1.3^{4}, 1.3 .9\right)$ & 4 \\
13 & $(2,2,3,8)$ & $(1,2,3,8)$ & $\left(1^{13}, 1.2^{6}, 1.3^{4}, 1.4 .8\right)$ & 4 \\
17 & $(2,3,3,8)$ & $(1,2,3,8)$ & $\left(1^{17}, 1.2^{8}, 1^{2} .3^{5}, 1.8^{2}\right)$ & 9 \\
17 & $(2,4,5,5)$ & $(1,2,4,5)$ & $\left(1^{17}, 1.2^{8}, 1.4^{4}, 1^{2} .5^{3}\right)$ & 8 \\
19 & $(2,3,4,8)$ & $(1,2,3,8)$ & $\left(1^{19}, 1.2^{9}, 1.3^{6}, 1.2 .8^{2}\right)$ & 10 \\
22 & $(2,2,3,7)$ & $(1,2,3,7)$ & $\left(1^{22}, 1^{2} .2^{10}, 1.3^{7}, 1.7^{3}\right)$ & 13 \\
29 & $(2,3,3,7)$ & $(1,2,3,7)$ & $\left(1^{29}, 1.2^{14}, 1^{2} .3^{9}, 1.7^{4}\right)$ & 14 \\
37 & $(2,3,7,7)$ & $(1,2,3,7)$ & $\left(1^{37}, 1.2^{18}, 1.3^{12}, 1^{2} .7^{5}\right)$ & 15 \\
\hline \hline
\end{tabular}

TABLE 2. Sporadic inclusions, corresponding passports, and number of realisations in Case 4.

Remark 4.1. 1) The complete list of ordered numerical solutions of (iv) in Lemma 3.6 include also the following two non-realisable solutions

- $N=4,(p, q, r, s)=(1,3, r, r),(k, l, m, n)=(1,2,3,2 r)$ with corresponding passport $\left(1^{4}, 2^{2}, 1.3,2^{2}\right)$ and

- $N=16,(p, q, r, s)=(1,3,7,7),(k, l, m, n)=(1,2,3,7)$ with corresponding passport $\left(1^{16}, 2^{8}, 1.3^{5}, 1^{2} .7^{2}\right)$.

2) In tables 1,2 and 3-8 (in the Appendix, corresponding to Cases 1-3), the passport is ordered according to $(k, l, m, n)$; since a permutation of $(k, l, m, n)$ corresponds to a permutation of the generators $x, y, z, w$ of $Q(k, l, m, n)$ and hence to a permutation of the passport $\left(\lambda\left(\pi_{x}\right), \lambda\left(\pi_{y}\right), \lambda\left(\pi_{z}\right), \lambda\left(\pi_{w}\right)\right)$. The 4 -tuples $(p, q, r, s)$ 
are independently ordered. For example lines 4 and 5 in Table 1

\begin{tabular}{|r|r|c|c|r|}
\hline \hline$N$ & $(p, q, r, s)$ & $(k, l, m, n)$ & Passport & $\#$ \\
\hline \hline 3 & $(1,2, r, 2 r)$ & $(1,2,3,2 r)$ & $\left(1^{3}, 1.2,3,1.2\right)$ & 1 \\
4 & $(2,2,3, s)$ & $(1,2,3,4 s)$ & $\left(1^{4}, 1^{2} .2,1.3,4\right)$ & 1 \\
\hline \hline
\end{tabular}

are reorderings (in the above sense) of the following lines

\begin{tabular}{|r|r|c|c|r|}
\hline \hline$N$ & $(p, q, r, s)$ & $(k, l, m, n)$ & Passport & $\#$ \\
\hline \hline 3 & $(2,1, r, 2 r)$ & $(1,2,3,2 r)$ & $\left(1^{3}, 1.2,3,1.2\right)$ & 1 \\
4 & $(3, s, 2,2)$ & $(1,3,4 s, 2)$ & $\left(1^{4}, 1.3,4,1^{2} .2\right)$ & 1 \\
\hline \hline
\end{tabular}

\section{Curiosities And Remarks}

Example 5.1 (Inclusions realisable by different passports).

1. Let $N=12$ and $(p, q, r, s)=(2,2,5,5)$. In Case 3 (see Table 8 in the Appendix), setting $(k, l, m, n)=(1,2,4,5)$ we get a numerical solution with associated passport $\left(1^{12}, 2^{5}, 2^{2} .4^{2}, 1^{2} .5^{2}\right)$, while setting $(k, l, m, n)=(1,4,2,5)$ we get a numerical solution with associated passport $\left(1^{12}, 4^{3}, 1^{2} \cdot 2^{5}, 1^{2} .5^{2}\right)$. Both give rise to the inclusion $Q(2,2,5,5)<_{12} Q(1,2,4,5) \cong Q(1,4,2,5)$ (Lemma 2.2) since there are constellations with both passports. More precisely, according to Table 8 , there are 3 non-isomorphic 4 -constellations with passport $\left(1^{12}, 2^{5}, 2^{2} .4^{2}, 1^{2} .5^{2}\right)$ and 6 nonisomorphic 4-constellations with passport $\left(1^{12}, 4^{3}, 1^{2} \cdot 2^{5}, 1^{2} .5^{2}\right)$.

2. Another example is given by the numerical solution $N=10,(p, q, r, s)=$ $(2,2,4,4)$ and $(k, l, m, n)=(1,5,2,4)$, which is at the same time a numerical solution for (iv) in Case 1, with associated passport $\left(1^{10}, 5^{2}, 2^{5}, 1^{2} .2^{2} .4\right)$, and a numerical solution for (iv) in Case 3, with associated passport $\left(1^{10}, 5^{2}, 1^{2} .2^{4}, 1^{2} .4^{2}\right)$. The existence of an inclusion $Q(2,2,4,4)<_{10} \Delta(2,4,5) \cong Q(1,5,2,4)$ (Lemma $2.2)$ is guaranteed by the existence of a hypermap with passport $\left(5^{2}, 2^{5}, 1^{2} .2^{2} .4\right)$ or $\left(5^{2}, 1^{2} \cdot 2^{4}, 1^{2} \cdot 4^{2}\right)$. According to Table 4 (Appendix), there are two constellations with the fist passport and five with the second passport, given by the spherical hypermaps in the following two Figures.

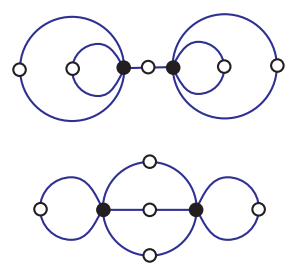

Figure 2: Hypermaps with passport $\left(5^{2}, 2^{5}, 1^{2} .2^{2} .4\right)$.

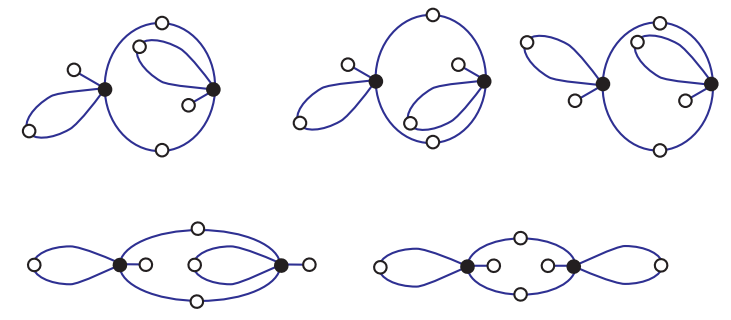

Figure 3: Hypermaps with passport $\left(5^{2}, 1^{2} \cdot 2^{4}, 1^{2} \cdot 4^{2}\right)$.

According to Tables 1-8, there is no other inclusion which is realisable by different passports.

Example 5.2 (A passport giving rise to different inclusions). The same spherical hypermap may prove the existence of different generalised quadrangle group inclusions. The following figure shows a hypermap which proves the existence of three distinct families of inclusions of index 4. These three families obviously have the same corresponding passport (up to permutation, according to Lemma $2.2)$, which in this case is $\left(4,1^{2} .2,1.3\right)$ : 


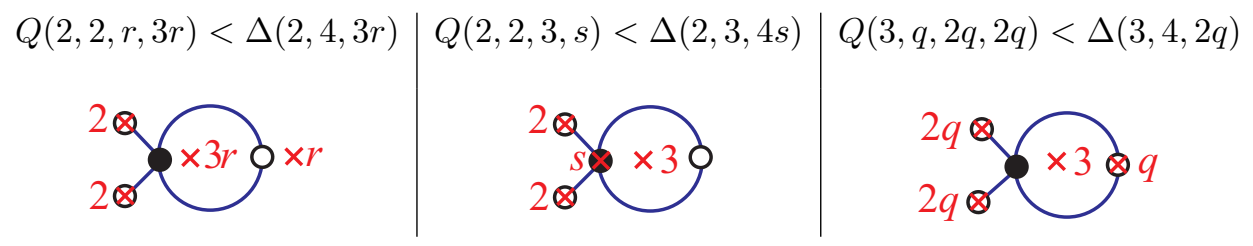

Figure 3: Three distinct families of inclusions with the same passport.

One can distinguish the three different inclusions by adding to the picture the orders of the subgroup generators as branched indices (at branched points, corresponding to those cycles of $\pi\left(x_{i}\right)$ of length less than $\left|x_{i}\right|$ mentioned in Proposition 3.2). In doing so we get three (representations of) distinct dessins d'enfant, as defined in [4].

Remark 5.3 (Maximal chains of inclusions). According to [13], for any maximal chain of inclusions ${ }^{3}$ of triangle groups $\Delta_{1}<\Delta_{2}<\cdots<\Delta_{t}$ one has that $\Delta_{t}$ is one of $\Delta(2,2 q, r), \Delta(3,3, q), \Delta(2,3,2 r), \Delta(2,3,3 r)$ or $\Delta(2,3,7)$.

We claim that the same assertion is true for maximal chains of inclusions of generalised quadrangle groups. In fact, from Tables 1-8 we conclude that for any inclusion $P<Q$ we have that $Q$ is a triangle group or $P<Q$ is one of the following inclusions:

$$
Q(p, p, r, r)<Q(2,2, p, r) \quad \text { or } \quad Q(p, p, p, p)<Q(2,2,2, p) .
$$

Remarking that any group in the 1-parametric family $Q(2,2,2, p)$ is isomorphic to a group of the 2-parametric family $Q(2,2, p, r)$ and that $Q(2,2, p, r)<\Delta(2,2 p, 2 r)$ (see first line of Table 1), our claim follows.

\footnotetext{
${ }^{3} \Delta_{t}<\Delta \Rightarrow \Delta_{t} \cong \Delta$
} 
Appendix: Tables of inclusions for the Cases 1-3.

\begin{tabular}{|r|r|c|c|r|}
\hline \hline$N$ & $(p, q, r, s)$ & $(k, l, m, n)$ & Passport & $\#$ \\
\hline \hline 4 & $(p, p, p, p)$ & $(1,4,4, p)$ & $\left(1^{4}, 4,4,1^{4}\right)$ & 1 \\
4 & $(p, p, p, p)$ & $(2,2,2, p)$ & $\left(2^{2}, 2^{2}, 2^{2}, 1^{4}\right)$ & 1 \\
6 & $(p, 3 p, 3 p, 3 p)$ & $(1,2,6,3 p)$ & $\left(1^{6}, 2^{3}, 6,1^{3} .3\right)$ & 1 \\
6 & $(p, p, 2 p, 2 p)$ & $(1,2,6,2 p)$ & $\left(1^{6}, 2^{3}, 6,1^{2} .2^{2}\right)$ & 1 \\
6 & $(p, p, 2 p, 2 p)$ & $(1,3,3,2 p)$ & $\left(1^{6}, 3^{2}, 3^{2}, 1^{2} .2^{2}\right)$ & 1 \\
8 & $(p, 2 p, 4 p, 4 p)$ & $(1,2,4,4 p)$ & $\left(1^{8}, 2^{4}, 4^{2}, 1^{2} .2 .4\right)$ & 1 \\
8 & $(p, p, 3 p, 3 p)$ & $(1,2,4,3 p)$ & $\left(1^{8}, 2^{4}, 4^{2}, 1^{2} .3^{2}\right)$ & 1 \\
8 & $(p, p, p, p)$ & $(1,2,4,2 p)$ & $\left(1^{8}, 2^{4}, 4^{2}, 2^{4}\right)$ & 1 \\
12 & $(p, 9 p, 9 p, 9 p)$ & $(1,2,3,9 p)$ & $\left(1^{12}, 2^{6}, 3^{4}, 1^{3} .9\right)$ & 1 \\
12 & $(p, 4 p, 8 p, 8 p)$ & $(1,2,3,8 p)$ & $\left(1^{12}, 2^{6}, 3^{4}, 1^{2} .2 .8\right)$ & 1 \\
12 & $(p, p, 5 p, 5 p)$ & $(1,2,3,5 p)$ & $\left(1^{12}, 2^{6}, 3^{4}, 1^{2} .5^{2}\right)$ & 1 \\
12 & $(p, 2 p, 3 p, 6 p)$ & $(1,2,3,6 p)$ & $\left(1^{12}, 2^{6}, 3^{4}, 1.2 .3 .6\right)$ & 1 \\
12 & $(p, p, 2 p, 2 p)$ & $(1,2,3,4 p)$ & $\left(1^{12}, 2^{6}, 3^{4}, 2^{2} .4^{2}\right)$ & 1 \\
12 & $(p, p, p, p)$ & $(1,2,3,3 p)$ & $\left(1^{12}, 2^{6}, 3^{4}, 3^{4}\right)$ & 1 \\
\hline
\end{tabular}

TABLE 3. Families of inclusions, corresponding passports, and number of realisations in Case 1.

\begin{tabular}{|r|r|l|c|c|}
\hline \hline$N$ & $(p, q, r, s)$ & $(k, l, m, n)$ & Passport & $\#$ \\
\hline \hline 8 & $(4,4,4,4)$ & $(1,2,4,8)$ & $\left(1^{8}, 2^{4}, 1^{4} .4,8\right)$ & 1 \\
10 & $(3,3,3,3)$ & $(1,2,3,10)$ & $\left(1^{10}, 2^{5}, 1^{4} .3^{2}, 10\right)$ & 1 \\
10 & $(6,6,6,6)$ & $(1,2,5,6)$ & $\left(1^{10}, 2^{5}, 5^{2}, 1^{4} .6\right)$ & 1 \\
10 & $(2,2,4,4)$ & $(1,2,4,5)$ & $\left(1^{10}, 2^{5}, 1^{2} .2^{2} .4,5^{2}\right)$ & 2 \\
12 & $(4,4,4,4)$ & $(1,2,4,6)$ & $\left(1^{12}, 2^{6}, 1^{4} .4^{2}, 6^{2}\right)$ & 2 \\
12 & $(4,4,4,4)$ & $(1,3,3,4)$ & $\left(1^{12}, 3^{4}, 3^{4}, 1^{4} .4^{2}\right)$ & 1 \\
12 & $(2,6,6,6)$ & $(1,2,4,6)$ & $\left(1^{12}, 2^{6}, 4^{3}, 1^{3} .3 .6\right)$ & 1 \\
12 & $(3,3,6,6)$ & $(1,2,4,6)$ & $\left(1^{12}, 2^{6}, 4^{3}, 1^{2} .2^{2} .6\right)$ & 1 \\
16 & $(3,3,3,3)$ & $(1,2,3,8)$ & $\left(1^{16}, 2^{8}, 1^{4} .3^{4}, 8^{2}\right)$ & 3 \\
16 & $(6,6,6,6)$ & $(1,2,4,6)$ & $\left(1^{16}, 2^{8}, 4^{4}, 1^{4} .6^{2}\right)$ & 2 \\
18 & $(14,14,14,14)$ & $(1,2,3,14)$ & $\left(1^{18}, 2^{9}, 3^{6}, 1^{4} .14\right)$ & 1 \\
18 & $(4,12,12,12)$ & $(1,2,3,12)$ & $\left(1^{18}, 2^{9}, 3^{6}, 1^{3} .3 .12\right)$ & 1 \\
18 & $(2,10,10,10)$ & $(1,2,3,10)$ & $\left(1^{18}, 2^{9}, 3^{6}, 1^{3} .5 .10\right)$ & 1 \\
18 & $(6,6,12,12)$ & $(1,2,3,12)$ & $\left(1^{18}, 2^{9}, 3^{6}, 1^{2} .2^{2} .12\right)$ & 1 \\
20 & $(4,4,4,4)$ & $(1,2,4,5)$ & $\left(1^{20}, 2^{10}, 1^{4} .4^{4}, 5^{4}\right)$ & 3 \\
24 & $(5,5,5,5)$ & $(1,2,4,5)$ & $\left(1^{24}, 2^{12}, 4^{6}, 1^{4} .5^{4}\right)$ & 5 \\
24 & $(10,10,10,10)$ & $(1,2,3,10)$ & $\left(1^{24}, 2^{12}, 3^{8}, 1^{4} .10^{2}\right)$ & 3 \\
24 & $(1,7,7,7)$ & $(1,2,3,7)$ & $\left(1^{24}, 2^{12}, 3^{8}, 1^{3} .7^{3}\right)$ & 1 \\
24 & $(2,4,8,8)$ & $(1,2,3,8)$ & $\left(1^{24}, 2^{12}, 3^{8}, 1^{2} .2 .4 .8^{2}\right)$ & 1 \\
24 & $(4,4,4,4)$ & $(1,2,3,8)$ & $\left(1^{24}, 2^{12}, 3^{8}, 2^{4} .8^{2}\right)$ & 1 \\
28 & $(3,3,3,3)$ & $(1,2,3,7)$ & $\left(1^{28}, 2^{14}, 1^{4} .3^{8}, 7^{4}\right)$ & 5 \\
30 & $(4,4,8,8)$ & $(1,2,3,8)$ & $\left(1^{30}, 2^{15}, 3^{10}, 1^{2} .2^{2} .8^{3}\right)$ & 3 \\
36 & $(8,8,8,8)$ & $(1,2,3,8)$ & $\left(1^{36}, 2^{18}, 3^{12}, 1^{4} .8^{4}\right)$ & 4 \\
60 & $(7,7,7,7)$ & $(1,2,3,7)$ & $\left(1^{60}, 2^{30}, 3^{20}, 1^{4} .7^{8}\right)$ & 6 \\
\hline \hline
\end{tabular}

TABLE 4. Sporadic inclusions, corresponding passports, and number of realisations in Case 1. 


\begin{tabular}{|r|r|l|c|c|}
\hline \hline$N$ & $(p, q, r, s)$ & $(k, l, m, n)$ & Passport & $\#$ \\
\hline \hline 3 & $(p, q, q, q)$ & $(1,3,3 p, q)$ & $\left(1^{3}, 3,3,1^{3}\right)$ & 1 \\
3 & $(1, q, q, q)$ & $(1,3,3, q)$ & $\left(1^{3}, 3,3,1^{3}\right)$ & 1 \\
4 & $(3, q, 2 q, 2 q)$ & $(1,3,4,2 q)$ & $\left(1^{4}, 1.3,4,1^{2} .2\right)$ & 1 \\
4 & $(p, q, 2 q, 2 q)$ & $(1,2,4 p, 2 q)$ & $\left(1^{4}, 2^{2}, 4,1^{2} .2\right)$ & 1 \\
4 & $(1, q, 2 q, 2 q)$ & $(1,2,4,2 q)$ & $\left(1^{4}, 2^{2}, 4,1^{2} .2\right)$ & 1 \\
5 & $(2, q, 3 q, 3 q)$ & $(1,2,5,3 q)$ & $\left(1^{5}, 1.2^{2}, 5,1^{2} .3\right)$ & 1 \\
5 & $(2, q, q, 2 q)$ & $(1,2,5,2 q)$ & $\left(1^{5}, 1.2^{2}, 5,1.2^{2}\right)$ & 1 \\
6 & $(p, 3,3,3)$ & $(1,2,6 p, 3)$ & $\left(1^{6}, 2^{3}, 6,1^{3} .3\right)$ & 1 \\
6 & $(5, q, 4 q, 4 q)$ & $(1,2,5,4 q)$ & $\left(1^{6}, 2^{3}, 1.5,1^{2} .4\right)$ & 1 \\
6 & $(1, q, 4 q, 4 q)$ & $(1,2,3,4 q)$ & $\left(1^{6}, 2^{3}, 3^{2}, 1^{2} .4\right)$ & 1 \\
6 & $(5,2 q, 3 q, 6 q)$ & $(1,2,5,6 q)$ & $\left(1^{6}, 2^{3}, 1.5,1.2 .3\right)$ & 1 \\
6 & $(2,2 q, 3 q, 6 q)$ & $(1,2,4,6 q)$ & $\left(1^{6}, 2^{3}, 2.4,1.2 .3\right)$ & 1 \\
6 & $(1, q, q, q)$ & $(1,2,3,2 q)$ & $\left(1^{6}, 2^{3}, 3^{2}, 2^{3}\right)$ & 1 \\
9 & $(2, q, 7 q, 7 q)$ & $(1,2,3,7 q)$ & $\left(1^{9}, 1.2^{4}, 3^{3}, 1^{2} .7\right)$ & 1 \\
9 & $(2, q, 3 q, 6 q)$ & $(1,2,3,6 q)$ & $\left(1^{9}, 1.2^{4}, 3^{3}, 1.2 .6\right)$ & 1 \\
9 & $(2,3 q, 5 q, 15 q)$ & $(1,2,3,15 q)$ & $\left(1^{9}, 1.2^{4}, 3^{3}, 1.3 .5\right)$ & 1 \\
9 & $(2,3 q, 4 q, 6 q)$ & $(1,2,3,12 q)$ & $\left(1^{9}, 1.2^{4}, 3^{3}, 2.3 .4\right)$ & 1 \\
10 & $(3, q, 8 q, 8 q)$ & $(1,2,3,8 q)$ & $\left(1^{10}, 2^{5}, 1.3^{3}, 1^{2} .8\right)$ & 1 \\
10 & $(3,2 q, 7 q, 14 q)$ & $(1,2,3,14 q)$ & $\left(1^{10}, 2^{5}, 1.3^{3}, 1.2 .7\right)$ & 1 \\
10 & $(3,4 q, 5 q, 20 q)$ & $(1,2,3,20 q)$ & $\left(1^{10}, 2^{5}, 1.3^{3}, 1.4 .5\right)$ & 1 \\
10 & $(3,6 q, 10 q, 15 q)$ & $(1,2,3,30 q)$ & $\left(1^{10}, 2^{5}, 1.3^{3}, 2.3 .5\right)$ & 1 \\
\hline \hline
\end{tabular}

TABLE 5. Families of inclusions, corresponding passports, and number of realisations in Case 2. 


\begin{tabular}{|c|c|c|c|c|}
\hline$N$ & $(p, q, r, s)$ & $(k, l, m, n)$ & Passport & $\#$ \\
\hline 5 & $\overline{(\overline{(2,2,2,4)}}$ & (1,2,4,5) & $\left({ }^{\left(1^{5}, 1^{3} .2,1.4,5\right)}\right.$ & 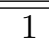 \\
\hline 6 & $(3,3,3,5)$ & $(1,3,3,5)$ & $\left(1^{6}, 3^{2}, 1^{3} .3,1.5\right)$ & 1 \\
\hline 6 & $(2,3,3,3)$ & $(1,3,3,4)$ & $\left(1^{6}, 3^{2}, 1^{3} .3,2.4\right)$ & 1 \\
\hline 7 & $(2,2,2,3)$ & $(1,2,3,7)$ & $\left(1^{7}, 1^{3} .2^{2}, 1.3^{2}, 7\right)$ & 2 \\
\hline 7 & $(2,4,4,4)$ & $(1,2,4,7)$ & $\left(1^{7}, 1.2^{3}, 1^{3} .4,7\right)$ & 1 \\
\hline 8 & $(5,5,5,7)$ & $(1,2,5,7)$ & $\left(1^{8}, 2^{4}, 1^{3} .5,1.7\right)$ & 1 \\
\hline 8 & $(2,4,4,7)$ & $(1,2,4,7)$ & $\left(1^{8}, 2^{4}, 1^{2} .2 .4,1.7\right)$ & 2 \\
\hline 8 & $(2,3,4,4)$ & $(1,2,4,6)$ & $\left(1^{8}, 2^{4}, 1^{2} .2 .4,2.6\right)$ & 1 \\
\hline 8 & $(1,3,3,7)$ & $(1,2,3,7)$ & $\left(1^{8}, 2^{4}, 1^{2} .3^{2}, 1.7\right)$ & 1 \\
\hline 9 & $(2,2,2,8)$ & $(1,2,3,8)$ & $\left(1^{9}, 1^{3} \cdot 2^{3}, 3^{3}, 1.8\right)$ & 2 \\
\hline 9 & $(2,3,3,3)$ & $(1,2,3,9)$ & $\left(1^{9}, 1.2^{4}, 1^{3} .3^{2}, 9\right)$ & 2 \\
\hline 9 & $(3,3,3,4)$ & $(1,3,3,4)$ & $\left(1^{9}, 3^{3}, 1^{3} \cdot 3^{2}, 1.4^{2}\right)$ & 1 \\
\hline 12 & $(3,3,3,11)$ & $(1,2,3,11)$ & $\left(1^{12}, 2^{6}, 1^{3} \cdot 3^{3}, 1.11\right)$ & 2 \\
\hline 12 & $(3,3,3,5)$ & $(1,2,3,10)$ & $\left(1^{12}, 2^{6}, 1^{3} .3^{3}, 2.10\right)$ & 1 \\
\hline 12 & $(3,3,3,3)$ & $(1,2,3,9)$ & $\left(1^{12}, 2^{6}, 1^{3} .3^{3}, 3.9\right)$ & 1 \\
\hline 12 & $(2,3$, & $(1,2,3,8)$ & $\left(1^{12}, 2^{6}, 1^{3} \cdot 3^{3}, 4.8\right)$ & 1 \\
\hline 12 & $(1,9,9,9)$ & $(1,2,3,9)$ & $\left(1^{12}, 2^{6}, 3^{4}, 1^{3} .9\right)$ & 1 \\
\hline 12 & $(1,4,8,8)$ & $(1,2,3,8)$ & $\left(1^{12}, 2^{6}, 3^{4}, 1^{2} .2 .8\right)$ & 1 \\
\hline 15 & $(2,2,2,7)$ & $(1,2,3,7)$ & $\left(1^{15}, 1^{3} \cdot 2^{6}, 3^{5}, 1.7^{2}\right)$ & 3 \\
\hline 15 & $(2,4,4,4)$ & $(1,2,4,5)$ & $\left(1^{15}, 1.2^{7}, 1^{3} \cdot 4^{3}, 5^{3}\right)$ & 1 \\
\hline 15 & $(2,12,12,12)$ & $(1,2,3,12)$ & $\left(1^{15}, 1.2^{7}, 3^{5}, 1^{3} .12\right)$ & 2 \\
\hline 15 & $(2,5,5,10)$ & $(1,2,3,10)$ & $\left(1^{15}, 1.2^{7}, 3^{5}, 1.2^{2} .10\right)$ & 1 \\
\hline 15 & $(2,2,4,8)$ & $(1,2,3,8)$ & $\left(1^{15}, 1.2^{7}, 3^{5}, 1.2 .4 .8\right)$ & 2 \\
\hline 16 & $(3,13,13,13)$ & $(1,2,3,13)$ & $\left(1^{16}, 2^{8}, 1.3^{5}, 1^{3} .13\right)$ & 2 \\
\hline 16 & $(2,4,4,5)$ & $(1,2,4,5)$ & $\left(1^{16}, 2^{8}, 1^{2} \cdot 2 \cdot 4^{3}, 1.5^{3}\right)$ & 3 \\
\hline 16 & $(3,6,12,12)$ & $(1,2,3,12)$ & $\left(1^{16}, 2^{8}, 1.3^{5}, 1^{2} \cdot 2.12\right)$ & 3 \\
\hline 16 & $(3,3,3,9)$ & $(1,2,3,9)$ & $\left(1^{16}, 2^{8}, 1.3^{5}, 1.3^{2} .9\right)$ & 1 \\
\hline 18 & $(3,3,3,4)$ & $(1,2,3,8)$ & $\left(1^{18}, 2^{9}, 1^{3} \cdot 3^{5}, 2.8^{2}\right)$ & 1 \\
\hline 21 & $(2,3,3,3)$ & $(1,2,3,7)$ & $\left(1^{21}, 1.2^{10}, 1^{3} \cdot 3^{6}, 7^{3}\right)$ & 4 \\
\hline 21 & $(2,9,9,9)$ & $(1,2,3,9)$ & $\left(1^{21}, 1.2^{10}, 3^{7}, 1^{3} .9^{2}\right)$ & 2 \\
\hline 24 & $(1,7,7,7)$ & $(1,2,3,7)$ & $\left(1^{24}, 2^{12}, 3^{8}, 1^{3} \cdot 7^{3}\right)$ & 1 \\
\hline 27 & $(2,8,8,8)$ & $(1,2,3,8)$ & $\left(1^{27}, 1.2^{13}, 3^{9}, 1^{3} .8^{3}\right)$ & 2 \\
\hline 28 & $(3,4,8,8)$ & $(1,2,3,8)$ & $\left(1^{28}, 2^{14}, 1.3^{9}, 1^{2} \cdot 2.8^{3}\right)$ & 2 \\
\hline 36 & $(3,3,3,7)$ & $(1,2,3,7)$ & $\left(1^{36}, 2^{18}, 1^{3} \cdot 3^{11}, 1.7^{5}\right)$ & 4 \\
\hline 45 & $(2,7,7,7)$ & $(1,2,3,7)$ & $\left(1^{45}, 1.2^{22}, 3^{15}, 1^{3} \cdot 7^{6}\right)$ & 2 \\
\hline 52 & $(3,7,7,7)$ & $(1,2,3,7)$ & $\left(1^{52}, 2^{26}, 1.3^{17}, 1^{3} .7^{7}\right)$ & 1 \\
\hline
\end{tabular}

TABLE 6. Sporadic inclusions, corresponding passports, and number of realisations in Case 2. 


\begin{tabular}{|r|r|l|c|c|}
\hline \hline$N$ & $(p, q, r, s)$ & $(k, l, m, n)$ & Passport & $\#$ \\
\hline \hline 2 & $(p, p, r, r)$ & $(2,2, p, r)$ & $\left(2,2,1^{2}, 1^{2}\right)$ & 1 \\
3 & $(p, 2 p, r, 2 r)$ & $(1,3,2 p, 2 r)$ & $\left(1^{3}, 3,1.2,1.2\right)$ & 1 \\
4 & $(2,2, r, 3 r)$ & $(1,2,4,3 r)$ & $\left(1^{4}, 1^{2} .2,4,1.3\right)$ & 1 \\
4 & $(2,2, r, r)$ & $(1,2,4,2 r)$ & $\left(1^{4}, 1^{2} .2,4,2^{2}\right)$ & 1 \\
4 & $(p, 3 p, r, 3 r)$ & $(1,2,3 p, 3 r)$ & $\left(1^{4}, 2^{2}, 1.3,1.3\right)$ & 1 \\
4 & $(p, p, r, r)$ & $(1,2,2 p, 2 r)$ & $\left(1^{4}, 2^{2}, 2^{2}, 2^{2}\right)$ & 1 \\
6 & $(2,2, r, 5 r)$ & $(1,2,3,5 r)$ & $\left(1^{6}, 1^{2} .2^{2}, 3^{2}, 1.5\right)$ & 1 \\
6 & $(2,2, r, 2 r)$ & $(1,2,3,4 r)$ & $\left(1^{6}, 1^{2} .2^{2}, 3^{2}, 2.4\right)$ & 1 \\
6 & $(2,2, r, r)$ & $(1,2,3,3 r)$ & $\left(1^{6}, 1^{2} .2^{2}, 3^{2}, 3^{2}\right)$ & 1 \\
6 & $(4,4, r, 5 r)$ & $(1,2,4,5 r)$ & $\left(1^{6}, 2^{3}, 1^{2} .4,1.5\right)$ & 1 \\
6 & $(4,4, r, r)$ & $(1,2,4,3 r)$ & $\left(1^{6}, 2^{3}, 1^{2} .4,3^{2}\right)$ & 1 \\
8 & $(3,3, r, 7 r)$ & $(1,2,3,7 r)$ & $\left(1^{8}, 2^{4}, 1^{2} .3^{2}, 1.7\right)$ & 1 \\
8 & $(3,3, r, 3 r)$ & $(1,2,3,6 r)$ & $\left(1^{8}, 2^{4}, 1^{2} .3^{2}, 2.6\right)$ & 1 \\
8 & $(3,3, r, r)$ & $(1,2,3,4 r)$ & $\left(1^{8}, 2^{4}, 1^{2} .3^{2}, 4^{2}\right)$ & 1 \\
\hline
\end{tabular}

TABLE 7. Families of inclusions, corresponding passports, and number of realisations in Case 3 . 


\begin{tabular}{|c|c|c|c|c|}
\hline$N$ & $(p, q, r, s)$ & $(k, l, m, n)$ & Passport & $\#$ \\
\hline 5 & $\overline{(\overline{(3,3,3,3)}}$ & (1,3,3,5) & 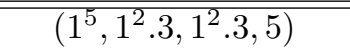 & $\overline{1} 1$ \\
\hline 6 & $(2,2,4,4)$ & $(1,2,4,6)$ & $\left(1^{6}, 1^{2} \cdot 2^{2}, 1^{2} \cdot 4,6\right)$ & 2 \\
\hline 6 & $(4,4,4,4)$ & $(1,3,4,4)$ & $\left(1^{6}, 3^{2}, 1^{2} \cdot 4,1^{2} .4\right)$ & 2 \\
\hline 8 & $(2,2,3,3)$ & $(1,2,3,8)$ & $\left(1^{8}, 1^{2} \cdot 2^{3}, 1^{2} \cdot 3^{2}, 8\right)$ & 4 \\
\hline 8 & $(2,2,6,6)$ & $(1,2,4,6)$ & $\left(1^{8}, 1^{2} \cdot 2^{3}, 4^{2}, 1^{2} .6\right)$ & 3 \\
\hline 8 & $(3,3,3,3)$ & $(1,3,3,4)$ & $\left(1^{8}, 1^{2} \cdot 3^{2}, 1^{2} \cdot 3^{2}, 4^{2}\right)$ & 3 \\
\hline 8 & $(6,6,6,6)$ & $(1,2,6,6)$ & $\left(1^{8}, 2^{4}, 1^{2} \cdot 6,1^{2} \cdot 6\right)$ & 2 \\
\hline 9 & $(1,2,7,7)$ & $(1,2,3,7)$ & $\left(1^{9}, 1.2^{4}, 3^{3}, 1^{2} .7\right)$ & 1 \\
\hline 10 & $(2,2,4,4)$ & $(1,2,4,5)$ & $\left(1^{10}, 1^{2} \cdot 2^{4}, 1^{2} \cdot 4^{2}, 5^{2}\right)$ & 5 \\
\hline 10 & $(4,4,8,8)$ & $(1,2,4,8)$ & $\left(1^{10}, 2^{5}, 1^{2} \cdot 4^{2}, 1^{2} .8\right)$ & 3 \\
\hline 10 & $(2,4,4,6)$ & $(1,2,4,6)$ & $\left(1^{10}, 2^{5}, 1^{2} .4^{2}, 1.3 .6\right)$ & 3 \\
\hline 10 & $(3,3,4$ & $(1,2,4$, & $\left(1^{10}, 2^{5}, 1^{2} \cdot 4^{2}, 2^{2} \cdot 6\right)$ & 1 \\
\hline 10 & $(1,3,8,8)$ & $(1,2,3,8)$ & $\left(1^{10}, 2^{5}, 1.3^{3}, 1^{2} .8\right)$ & 1 \\
\hline 12 & $(2,2,5,5)$ & $(1,2,4$, & $\left(1^{12}, 1^{2} \cdot 2^{5}, 4^{3}, 1^{2} \cdot 5^{2}\right)$ & 6 \\
\hline 12 & $(2,2,10,10)$ & $(1,2,3,10)$ & $\left(1^{12}, 1^{2} \cdot 2^{5}, 3^{4}, 1^{2} .10\right)$ & 4 \\
\hline 12 & $(2,2,4,4)$ & $(1,2,3$ & $\left(1^{12}, 1^{2} \cdot 2^{5}, 3^{4}, 2^{2} .8\right)$ & 1 \\
\hline 12 & $(5,5,5,5)$ & $(1,2,5,5)$ & $\left(1^{12}, 2^{6}, 1^{2} \cdot 5^{2}, 1^{2} \cdot 5^{2}\right)$ & 5 \\
\hline 12 & $(2,2,5,5)$ & $(1,2,4,5)$ & $\left(1^{12}, 2^{6}, 2^{2} \cdot 4^{2}, 1^{2} \cdot 5^{2}\right)$ & 3 \\
\hline 14 & $(2,2,3,3)$ & $(1,2,3$, & $\left(1^{14}, 1^{2} \cdot 2^{6}, 1^{2} \cdot 3^{4}, 7^{2}\right)$ & 9 \\
\hline 14 & $(3,3,12,12)$ & $(1,2,3,12)$ & $\left(1^{14}, 2^{7}, 1^{2} \cdot 3^{4}, 1^{2} \cdot 12\right)$ & 4 \\
\hline 14 & $(3,3,5,5)$ & $(1,2,3,10)$ & $\left(1^{14}, 2^{7}, 1^{2} \cdot 3^{4}, 2^{2} .10\right)$ & 1 \\
\hline 14 & $(2,3,3,4)$ & $(1,2,3,8)$ & $\left(1^{14}, 2^{7}, 1^{2} \cdot 3^{4}, 2.4 .8\right)$ & 2 \\
\hline 14 & $(4,4,6,6)$ & $(1,2,4,6)$ & $\left(1^{14}, 2^{7}, 1^{2} \cdot 4^{3}, 1^{2} \cdot 6^{2}\right)$ & 5 \\
\hline 18 & $(2,2,8,8)$ & $(1,2,3,8)$ & $\left(1^{18}, 1^{2} \cdot 2^{8}, 3^{6}, 1^{2} \cdot 8^{2}\right)$ & 8 \\
\hline 20 & $(3,3,9,9)$ & $(1,2,3,9)$ & $\left(1^{20}, 2^{10}, 1^{2} \cdot 3^{6}, 1^{2} .9^{2}\right)$ & 9 \\
\hline 20 & $(3,3,4,4)$ & $(1,2,3,8)$ & $\left(1^{20}, 2^{10}, 1^{2} \cdot 3^{6}, 2^{2} \cdot 8^{2}\right)$ & 4 \\
\hline 22 & $(4,4,5,5)$ & $(1,2,4,5)$ & $\left(1^{22}, 2^{11}, 1^{2} \cdot 4^{5}, 1^{2} \cdot 5^{4}\right)$ & 5 \\
\hline 26 & $(3,3,8,8)$ & $(1,2,3,8)$ & $\left(1^{26}, 2^{13}, 1^{2} \cdot 3^{8}, 1^{2} \cdot 8^{3}\right)$ & 5 \\
\hline 30 & $(2,2,7,7)$ & $(1,2,3,7)$ & $\left(1^{30}, 1^{2} \cdot 2^{14}, 3^{10}, 1^{2} \cdot 7^{4}\right)$ & 12 \\
\hline 44 & $(3,3,7,7)$ & $(1,2,3,7)$ & $\left(1^{44}, 2^{22}, 1^{2} \cdot 3^{14}, 1^{2} \cdot 7^{6}\right)$ & 16 \\
\hline
\end{tabular}

TABLE 8. Sporadic inclusions, corresponding passports, and number of realisations in Case 3 .

\section{Remarks (to Tables 1-8).}

1. The same triangle group inclusion can appear in different tables (Cases) since $p=1$ divides any integer.

2. If a triangle group inclusion appears in different cases, it appears with the same passport, since any triangle group inclusion has only one realisation. (Compare with Examples 5.1, where we give an inclusion appearing in different cases with different passports).

3. Some triangle group inclusions are particular instances of families of generalised quadrangle group inclusions. We did'nt eliminate them to emphasize the triangle group inclusions.

The following table gives all the details stated in the above remarks. 


\begin{tabular}{|c|c|c|c|c|}
\hline label & index & $\Delta$-inclusion & "corresponding $Q$-inclusion $(\mathrm{s})$ & Cases \\
\hline (a) & 2 & 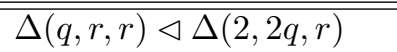 & $\overline{Q Q(p, q, r, r) \triangleleft \Delta(2 p, 2 q, r) \text { for } p=1}$ & 4 \\
\hline (b) & 3 & $\Delta(q, q, q) \triangleleft \Delta(3,3, q)$ & $Q(p, q, q, q) \triangleleft \Delta(3,3 p, q)$ for $p=1$ & 2 \\
\hline (c) & 6 & $\Delta(q, q, q) \triangleleft \Delta(2,3,2 q)$ & no correspondence & 2 \\
\hline (A) & 24 & $\Delta(7,7,7)<\Delta(2,3,7)$ & no correspondence & 1,2 \\
\hline (B) & 9 & $\Delta(2,7,7)<\Delta(2,3,7)$ & $Q(2, q, 7 q, 7 q)<\Delta(2$ & $2,3,4$ \\
\hline (C) & 8 & $\Delta(3,3,7)<\Delta(2,3,7)$ & $Q(3,3, r, 7)<\Delta(2,3,7 r)$ for $r=1$ & $2,3,4$ \\
\hline (D) & 12 & $\Delta(4,8,8)<\Delta(2,3,8)$ & $Q(p, 4 p, 8 p, 8 p)<\Delta(2,3,8 p)$ for $p=1$ & 1,2 \\
\hline (E) & 10 & $8)<\Delta(2$ & f) for $q=1$ & $2,3,4$ \\
\hline (F) & 12 & $\Delta(9,9,9)<\Delta(2,3,9)$ & $Q(p, 9 p, 9 p, 9 p)<\Delta(2,3,9 p)$ for $p=1$ & 1,2 \\
\hline \multirow[t]{2}{*}{ (G) } & 6 & $\Delta(4,4,5)<\Delta(2,4,5)$ & $Q(5, q, 4 q, 4 q)<\Delta(2,5,4 q)$ for $q=1$ or & \\
\hline & & & $Q(4$, & $2,3,4$ \\
\hline$(\mathrm{H})$ & 6 & $q, 4 q)<\Delta(2,3,4 q)$ & ondence & 2 \\
\hline (I) & 4 & $\Delta(q, 2 q, 2 q)<\Delta(2,4,2 q)$ & $Q(p, q, 2 q, 2 q)<\Delta(2,4 p, 2 q)$ for $p=1$ & 2 \\
\hline$(\mathrm{J})$ & 4 & $\Delta(3, r, 3 r)<\Delta(2,3,3 r)$ & $Q(p, 3 p, r, 3 r)<\Delta(2,3 p, 3 r)$ for $p=1$ & 3,4 \\
\hline \multirow[t]{2}{*}{$(\mathrm{K})$} & 3 & $\Delta(2, r, 2 r)<\Delta(2,3,2 r)$ & $Q(2, q, r, 2 r)<\Delta(2,3 q, 2 r)$ for $q=1$ or & \\
\hline & & & $Q(p, 2 p, r, 2 r)<\Delta(3,2 p, 2 r)$ for $p=1$ & 3,4 \\
\hline
\end{tabular}

TABLE 9. Triangle group inclusions as particular instances of families of generalised quadrangle group inclusions and respective cases.

\section{ACKNOWLEDGMENTS}

This work was supported by the Research and Development Cooperation project Slovakia - Portugal, ID number: SK-PT-NEWPROJECT-12522, supported by the portuguese side by the Portuguese Foundation for Science and Technology FCT (Fundação para a Ciência e a Tecnologia).

The work of the first two authors was partially supported by Portuguese funds through the CIDMA - Center for Research and Development in Mathematics and Applications (University of Aveiro) and FCT within project PEst-OE/MAT/UI4106/2014.

The work of of the third and the fourth author...

\section{REFERENCES}

[1] G. V. Bely̆̌, On Galois extensions of a maximal cyclotomic field, Math. USSR Isvestija 14 (1980), 247-256.

[2] W. Bosma, J. Cannon and C. Playoust, The Magma algebra system. I. The user language, J. Symbolic Comput., 24 (1997), 235265.

[3] A. Breda, D. A. Catalano, J. Karabáš and R. Nedela, Maps of Archimedean class and operations on dessins, Discrete Math. 338, Issue 10 (2015), 1814-1825.

[4] A. Breda, D. A. Catalano, J. Karabáš and R. Nedela, Census of Quadrangle Groups Inclusions, to appear in...

[5] A. Breda, D. A. Catalano, J. Karabáš and R. Nedela, Atlas of quadrangle groups inclusions, web page, http://www.savbb.sk/ karabas/science.html\#atlas.

[6] The GAP Group, GAP - Groups, Algorithms, and Programming, Version 4.7.6, 2014, (http://www.gap-system.org).

[7] E. Girondo, Multiply quasiplatonic Riemann surfaces, Exp. Math. 12, Issue 4 (2003), 463475.

[8] G. Jones, D. Singerman, Belyı functions, hypermaps and Galois groups, Bulletin of the London Mathematical Society 28 (1996), 561-590.

[9] G. A. Jones and D. Singerman, Complex Functions, an algebraic and geometric viewpoint, Cambrigde University Press, Cambridge 1988.

[10] S. Katok, Fuchsian groups, The University of Chicago Press, Chicago 1992.

[11] S. K. Lando and A. K. Zvonkin, Graphs on Surfaces and Their Applications, LowDimensional Topology II, Encyclopaedia of Mathematical Sciences, volume 141 (2003), Springer.

[12] D. Singerman, Subgroups of Fuchsian groups and finite permutation groups, Bull. London Math. Soc., 2 (1970), 319-323. 
[13] D. Singerman, Finitely maximal Fuchsian groups, J. London Math. Soc. (2), 6 (1972), 29-38.

[14] D. Singerman, Riemann Surfaces, Bely乞 Functions and Hypermaps, in Topics on Riemann Surfaces and Fuchsian Groups, London Mathematical Society, Lecture Note Series 287, Cambridge University Press, 2001.

[15] D. Singerman and R. I. Syddall, The Riemann surface of a uniform dessin, Beiträge Algebra Geom. 44 (2003), no. 2, 413-430.

[16] J. Wolfart, The 'Obvious' part of Belyi's Theorem and Riemann Surfaces with many automorphisms, pp. 97-112 in Geometric Galois Actions 1, ed. L. Schneps and P. Lochak, London Math. Soc. Lecture Notes Ser. 242, Cambridge University Press, Cambridge, 1997.

(A. Breda, D. Catalano) Departamento de Matemática, Universidade de Aveiro, 3810193 Aveiro, Portugal

E-mail address, A. Breda: breda@ua.pt

E-mail address, D. Catalano: domenico@ua.pt

(R. Nedela) Institute of Mathematics and Computer Science, Matej Bel University, 97401 Banská Bystrica, Slovakia

E-mail address, R. Nedela: nedela@savbb.sk

(J. Karabáš) FAMnit, University of Primorska, Glagoljaška 8, 6000 Koper, Slovenia E-mail address, J. Karabáš: karabas@savbb.sk 